\title{
Complicaciones durante la esofagoscopía con o sin dilatación con globo y con anestesia general en pacientes pediátricos: estudio prospectivo y observacional \\ Complications during esophageal endoscopy with or without baloon dilation under general anesthesia in pediatric patients: a prospective and observational study
}

\author{
Dra. Pinar Kendigelen , Prof. Asoc. Dra. Ayse C. Tutuncu, Dr. Senol Emre, \\ Prof. Dra. Fatis Altindas ${ }^{a}$ y Prof. Dra. Guner Kaya ${ }^{a}$
}

\section{RESUMEN}

Antecedentes. Durante una esofagoscopía en niños, pueden ocurrir complicaciones durante la dilatación. Identificamos alteraciones cardiorrespiratorias durantela esofagoscopía con o sin dilatación con globo y las complicaciones posoperatorias, en niños con anestesia.

Métodos. Estudio prospectivo, observacional de procedimientos endoscópicos en niños de 0-16 años. Se dividieron en dos grupos: con endoscopía-dilatación (Grupo ED) y dilatación con globo por estenosis esofágica, y con endoscopía sin dilatación (Grupo E): endoscopía diagnóstica, esofagoscopía de control o escleroterapia.

Registramos parámetros hemodinámicos y ventilatorios y las complicaciones durante la endoscopía, la dilatación y el seguimiento de dos horas en la sala de recuperación.

Resultados. Incluimos 102 procedimientos en 60 pacientes. La presión inspiratoria máxima (PIM) aumentó significativamente en ambos grupos $(p<0,001)$ y aumentó significativamente durante el procedimiento en el grupo ED $(p<0,001)$. La diferencia en la PIM antes y después de la endoscopía se correlacionó negativamente en ambos grupos. $\mathrm{Al}$ subdividir los grupos según el punto de corte de 2 años para comparar la diferencia en la PIM antes y después de la endoscopía, la PIM aumentó de manera estadísticamente significativa en ambos grupos en los menores de 2 años. En el grupo ED, la frecuencia cardíaca aumentó estadísticamente significativa $(p<0,001)$.

Conclusión. Durante la endoscopía, la PIM aumentó en niños con o sin dilatación con globo, especialmente en el grupo ED, y fue mayor en los niños más pequeños. Es necesario observar y tratar atentamente las complicaciones cardiorrespiratorias graves durante la dilatación con globo y con anestesia general.

Palabras clave: niños, endoscopía, dilatación, anestesia.

Conflicto de intereses:

Ninguno que declarar.

Recibido: 30-11-2016

Aceptado: 4-9-2017 http:/ / dx.doi.org/10.5546/aap.2018.98 Texto completo en inglés: http:/ / dx.doi.org/10.5546/ aap.2018.eng.98
Cómo citar: Kendigelen P, Tutuncu AC, Cedillo Emre S, et al. Complicaciones durante la esofagoscopía con o sin dilatación con globo y con anestesia general en pacientes pediátricos: estudio prospectivo y observacional. Arch Argent Pediatr 2018;116(2):98-104.

\section{INTRODUCCIÓN}

La esofagoscopía se realiza por diversos motivos en el grupo etario pediátrico. La esofagoscopía con fines diagnósticos, para la aplicación de inyecciones de agentes esclerosantes en los casos de hipertensión portal o solo con fines de control es relativamente menos complicada. Sin embargo, la dilatación con globo aplicada en la estenosis esofágica se asocia con diversas complicaciones, en particular en los grupos etarios más pequeños.

En los niños, se observa estenosis esofágica debido a corrección de fístula traqueoesofágica o esofagitis corrosiva tras la ingesta accidental de sustancias cáusticas, entre otras causas. El tratamiento de la estenosis esofágica se administra en intervalos que varían según la gravedad de la afección e implica la dilatación endoscópica con globo y con anestesia general. El grado de la lesión esofágica varía conforme al $\mathrm{pH}$ de la sustancia ingerida y la gravedad de la erosión y la ulceración del esófago. ${ }^{1}$ Es común observar desnutrición, retraso del crecimiento y problemas pulmonares causados por la aspiración crónica en los pacientes sometidos a una dilatación. En la bibliografía se encuentran datos 
limitados acerca de las complicaciones y los problemas relacionados con el manejo anestésico de los procedimientos endoscópicos, en especial en los pacientes sometidos a dilatación esofágica. ${ }^{2-4}$ Durante la dilatación esofágica pueden ocurrir diferentes complicaciones como resultado de la tensión sobre las vías respiratorias y las estructuras vasculares.

Nuestro objetivo era identificar las alteraciones cardiorrespiratorias que se presentan durante la esofagoscopía con o sin dilatación con globo y con anestesia en los niños e investigar las complicaciones posoperatorias.

\section{MÉTODOS}

Este estudio de cohorte prospectivo y observacional fue aprobado por el consejo de ética de nuestra institución (no: 83045809/604.01/02/270718, 2 de diciembre de 2014) y se registró en clinicaltrials.gov (NCT 02512770). Los padres otorgaron su consentimiento informado por escrito.

Se incluyó a los pacientes (edad: de 0 a 16 años) que tenían un estado físico I-III según la clasificación de la Asociación Estadounidense de Anestesiólogos (American Society of Anesthesiologists, ASA) y que tenían planificada una esofagoscopía con anestesia general entre enero y junio de 2015. Se excluyó a los pacientes con una clasificación ASA IV y que tuvieron endoscopías para extraer cuerpos extraños. Se subdividió a los pacientes en dos grupos: el grupo con endoscopía-dilatación (Grupo ED) de los pacientes sometidos a dilatación con globo debido a estenosis esofágica y el grupo con endoscopía (Grupo E) sin dilatación de los pacientes con endoscopía por motivos diagnósticos, esofagoscopía de control o escleroterapia.

Todos los pacientes recibieron $0,05 \mathrm{mg} / \mathrm{kg}$ de midazolam por vía I.V. como medicación preanestésica antes de ingresar al quirófano y de 1 a $3 \mathrm{mg} / \mathrm{kg}$ de propofol, $0,6 \mathrm{mg} / \mathrm{kg}$ de rocuronio, $1 \mu \mathrm{g} / \mathrm{kg}$ de fentanilo para la inducción y sevoflurano, una mezcla de $\mathrm{O}_{2}$ / aire para el mantenimiento de la anestesia después del monitoreo estándar (electrocardiograma [ECG], presión arterial sistólica y diastólica [PAS y PAD] no invasiva y frecuencia cardíaca [FC], saturación periférica de oxígeno $\left[\mathrm{SpO}_{2}\right]$ y dióxido de carbono al final de la espiración $\left[\mathrm{EtCO}_{2}\right]$ ).

Se intubó a los pacientes con un tubo endotraqueal adecuado para su edad (tubo endotraqueal Bicakcilar, Estambul, Turquía) y se configuraron los parámetros ventilatorios en modo de ventilación controlada por presión (VCP) con volumen corriente en $8-10 \mathrm{ml} / \mathrm{kg}$, presión positiva al final de la espiración (PPFE) en $5 \mathrm{cmH}_{2} \mathrm{O}$ y EtCO $\mathrm{Et}_{2}$ en $30-35 \mathrm{mmHg}$. En caso de una disminución del $30 \%$ en el volumen corriente durante la endoscopía, se aumentaba la presión inspiratoria (Pinsp) al menos $1 \mathrm{cmH}_{2} \mathrm{O}$ por cambio en un esfuerzo para mantener un volumen corriente mínimo de $8 \mathrm{ml} / \mathrm{kg}$. La presión inspiratoria máxima (PIM) se determinó en $35 \mathrm{cmH}_{2} \mathrm{O}$ durante la ventilación. En caso de un aumento de la PIM por encima de $35 \mathrm{cmH}_{2} \mathrm{O}$, se disminuía la presión de insuflación del globo.

Las alteraciones en los parámetros hemodinámicos o ventilatorios y las complicaciones, si se producían, se observaron y registraron antes de la endoscopía, durante la endoscopía y la dilatación y durante el seguimiento de dos horas en la sala de recuperación postanestésica.

También se registraron la ubicación de la estenosis esofágica (superior, media distal) y la cantidad de pacientes con procedimientos de dilatación. Se investigó el pH de las sustancias cáusticas ingeridas de manera accidental.

\section{Procedimiento de dilatación}

La esofagoscopía se realizó con un endoscopio de fibra óptica (endoscopio pediátrico Olympus Exera2, Tokio, Japón) después de administrar la anestesia y la ventilación adecuadas para establecer el grado de la estenosis esofágica y la situación. Luego, se empujó un globo dilatador de esófago (Mediglob-Boston Scientific Corp, Massachusetts, EE. UU.) del calibre adecuado (8-18F y $10 \mathrm{~cm}$ de longitud) bajo radioscopía siguiendo la técnica de Seldinger y se incrementó el volumen del globo durante 5 minutos hasta distender la estenosis. Después del procedimiento, se supervisó y registró el diámetro de la estenosis ampliada con el endoscopio de fibra óptica.

\section{Análisis estadístico}

Se usó el programa SPSS 15.0 para Windows para los análisis estadísticos. Los datos estadísticos descriptivos se indicaron como cifra, y las variables numéricas, como media, mediana y desviación estándar (DE). Se realizó la prueba $t$ de Student para comparar dos grupos independientes con distribución normal. Se utilizó la prueba U de Mann-Whitney cuando las variables no tenían una distribución normal. Se emplearon la prueba t para muestras emparejadas para comparar la media de una variable dependiente en dos grupos y las pruebas de Wilcoxon y Friedman 
para las variables no paramétricas. Los análisis de subgrupos se realizaron con la prueba de Wilcoxon y se interpretaron con la corrección de Bonferroni. Se usó la correlación de Spearman para medir el nivel de asociación entre las variables no paramétricas. Un valor de $p<0,05$ se aceptó como estadísticamente significativo.

\section{RESULTADOS}

Se incluyó en el estudio a 68 pacientes; 8 de ellos no cumplieron los requisitos (3 pacientes tuvieron una endoscopía para extraer un cuerpo extraño y 5 pacientes tenían un estado físico IV según la clasificación de la ASA). En total, el estudio estuvo compuesto de 60 pacientes (Grupo ED=30, Grupo E=30). En el período del estudio, 52 de los procedimientos se realizaron con dilatación con globo y 40, sin dilatación (Figura 1). En el Grupo ED, se realizaron cinco dilataciones en dos pacientes, cuatro en un paciente, tres en cuatro pacientes y dos en tres pacientes. En el grupo de referencia, se realizaron 40 endoscopías de control, tres veces en cuatro pacientes y dos veces en dos pacientes.
La media de edad y peso de los pacientes del Grupo E fue mayor que en el Grupo ED (Tabla 1).

En el Grupo ED había 13 pacientes con atresia esofágica, 15 con esofagitis corrosiva y dos con esofagitis infecciosa (esofagitis micótica asociada con una función inmunitaria deficiente).

En el Grupo E, 20 pacientes tuvieron una endoscopía y recibieron escleroterapia para la hipertensión portal y ocho eran pacientes que se realizaban una endoscopía de control por primera vez tras la ingesta de una sustancia cáustica. En dos pacientes se realizó una endoscopía tras una cirugía de acalasia.

El $\mathrm{pH}$ de las sustancias cáusticas ingeridas era alcalino en el 80,9\% de los casos y ácido en el $19,1 \%$.

El valor medio de la PIM antes y después de la endoscopía no mostró diferencias estadísticas entre los grupos $(p=0,727 ; p=0,852)$ (Tabla 2). Sin embargo, se observó un aumento estadísticamente significativo en la PIM media en el Grupo ED durante el procedimiento $(p<0,001)$ (Tabla 2). Los valores de PIM aumentaron significativamente después de la endoscopía en

Figura 1. Diagrama de flujo

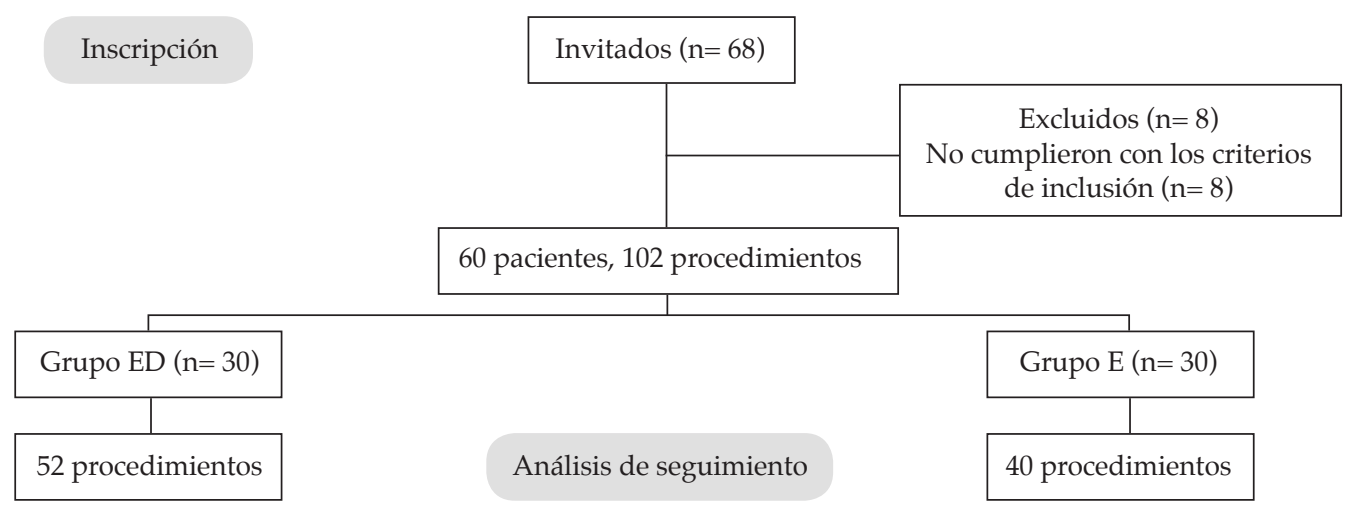

Tabla 1. Datos clínicos

\begin{tabular}{lcccc}
\hline & & $\begin{array}{c}\text { Grupo ED } \\
(\mathbf{n}=\mathbf{5 2} \text { procedimientos }) \\
\text { Media } \pm \text { DE }\end{array}$ & $\begin{array}{c}\text { Grupo E } \\
(\mathbf{n}=\mathbf{4 0} \text { procedimientos }) \\
\text { Media } \pm \text { DE }\end{array}$ & Valor $p$ \\
\hline Edad (años) & & $4,1 \pm 3,4$ & $9,5 \pm 5,4$ & $<0,001$ \\
& $\geq 2$ años & 28 & 33 & 0,004 \\
Peso (kg) & $<2$ años & 24 & $70,1 \pm 14,1$ & $<0,001$ \\
Estenosis (\%) & Proximal & $15,5 \pm 9,8$ & & \\
& Media inferior & 28 & & \\
\hline
\end{tabular}

Los datos se expresan como media \pm DE y cifra. 
ambos grupos (Tabla 2). Dicho aumento después de la endoscopía fue estadísticamente mayor en el grupo con dilatación $(p=0,044)$ (Tabla 2).

Se observó una correlación negativa de la diferencia en los valores de PIM antes y después de la endoscopía con la edad en ambos grupos (Grupo ED $r=-0,42, p=0,002$; Grupo E $r=-0,72$, $p<0,001)$. También se observó una correlación negativa de la diferencia en los valores de PIM antes de la endoscopía y la dilatación con la edad $(r=-0,49, p<0,001)$. Cuando se subdividieron los grupos según el punto de corte de 2 años de edad para comparar la diferencia en la PIM antes y después de la endoscopía, el aumento de la PIM fue estadísticamente mayor en ambos grupos en los niños menores de 2 años (Tabla 3). La comparación dentro del mismo grupo de los pacientes con dilatación mostró valores de PIM más elevados en los pacientes con estenosis esofágica proximal frente a los pacientes con estenosis media distal (Tabla 4). En cinco pacientes del Grupo ED, la PIM aumentó gravemente y la ventilación fue dificultosa hasta que se disminuyó la presión del globo.

No se observaron diferencias entre la media de PAS y de PAD entre ambos grupos. En el grupo ED, la FC aumentó de manera estadística durante el procedimiento $(p<0,001)$ (Tabla 5$)$. Se observó aparición repentina de la bradicardia en un paciente del Grupo ED durante el procedimiento de dilatación. Las complicaciones frecuentes en la sala de recuperación postanestésica fueron tos (10 pacientes: 8 en el Grupo ED, 2 en el Grupo E), agitación (6 pacientes: 5 en el Grupo ED, 1 en el Grupo E), taquicardia (3 pacientes en el Grupo E), náuseas y vómitos (8 pacientes: 7 en el Grupo ED, 1 en el Grupo E) y alteración de la voz (9 pacientes: 7 en el Grupo ED, 2 en el Grupo E). Ninguno de los pacientes presentó desaturación ni broncoespasmo.

Tabla 2. Presión inspiratoria máxima

\begin{tabular}{lccc}
\hline & $\begin{array}{c}\text { Grupo ED } \\
(\mathbf{n}=\mathbf{5 2} \text { procedimientos }) \\
\text { Media } \pm \mathrm{DE}\end{array}$ & $\begin{array}{c}\text { Grupo E } \\
(\mathbf{n}=\mathbf{4 0} \text { procedimientos }) \\
\text { Media } \pm \mathrm{DE}\end{array}$ & Valor $p$ \\
\hline $\begin{array}{l}\text { Antes de la } \\
\text { Endoscopía }\end{array}$ & $14,4 \pm 1,9$ & $14,7 \pm 2,3$ & 0,727 \\
Dilatación & $17,8 \pm 5,2$ & & 0,852 \\
Después de la & $15,3 \pm 2,2$ & $15,1 \pm 2,2$ & 0,044 \\
endoscopía & $<0,001$ & 0,004 & $0,35 \pm 0,66$ \\
Valor $p$ & $0,83 \pm 1,26$ & $0,35 \pm$ \\
\hline PIM & & & \\
\hline
\end{tabular}

$\Delta \mathrm{PIM}=$ diferencia antes y después de la endoscopía.

Los datos se expresan como media $\pm \mathrm{DE}$.

Tabla 3. Diferencia en la PIM antes y después de la endoscopía

\begin{tabular}{llcc}
\hline$\Delta$ PIM/ & Edad & Media \pm DE & Valor $p$ \\
\hline Grupo ED & $\geq 2$ años & $1,50 \pm 1,47$ & 0,001 \\
& $<2$ años & $0,25 \pm 0,65$ & 0,001 \\
Grupo E & $\geq 2$ años & $1,43 \pm 0,53$ & $<0,001$ \\
& $<2$ años & $0,12 \pm 0,42$ & $<0,001$ \\
\hline
\end{tabular}

$\Delta \mathrm{PIM}=$ diferencia antes y después de la endoscopía.

Los datos se expresan como media $\pm \mathrm{DE}$.

PIM: presión inspiratoria máxima.

TABLA 4. Presión inspiratoria máxima en la estenosis proximal y media inferior

\begin{tabular}{|c|c|c|c|}
\hline $\begin{array}{l}\text { Estenosis } \\
\text { PIM }\end{array}$ & $\begin{array}{c}\text { Proximal } \\
\text { Mediana [IIC] }\end{array}$ & $\begin{array}{c}\text { Media inferior } \\
\text { Mediana }[\mathrm{IIC}]\end{array}$ & Valor $p$ \\
\hline Diferencia entre D y AE & $3[2-7]$ & $1[0-1]$ & $<0,001$ \\
\hline Diferencia entre DE y AE & $1[1-2]$ & $0[0-0]$ & $<0,001$ \\
\hline
\end{tabular}

Los datos se expresan como mediana e intervalo intercuartílico (IIC). D: dilatación. AE: antes de la endoscopía. DE: después de la endoscopía. PIM: presión inspiratoria máxima. 


\section{DISCUSIÓN}

Si bien las dilataciones esofágicas son procedimientos quirúrgicos breves, los pacientes sometidos a estos procedimientos podrían presentar ciertos riesgos y complicaciones. Las causas principales de la estenosis esofágica en los niños incluyen la ingesta de sustancias cáusticas y la estenosis de la anastomosis / posquirúrgica en los recién nacidos con atresia esofágica. Otras etiologías incluyen estenosis congénita, esofagitis por reflujo, estenosis tras el tratamiento endoscópico de várices esofágicas, una sustancia extraña o una reacción a medicamentos orales y esofagitis infecciosa en niños con inmunodeficiencia. Los agentes con un $\mathrm{pH}<2 \mathrm{y}>12$ son sumamente corrosivos y causan complicaciones que varían de daño a perforación esofágica. ${ }^{5}$ Las lesiones esofágicas se clasifican como: sin lesión a grado 1, 2a, 2b, 3 (ulceración o perforación de grosor completo [transparietal]). ${ }^{6}$

La estenosis se produce en distintos niveles por esofagitis erosiva y desarrollo posterior de tejido fibroso. Frecuentemente, la estenosis se ubica en las porciones proximal y media del esófago. La ingesta de sustancias cáusticas es una situación altamente patológica que requiere un tratamiento complejo a largo plazo y que representa un deterioro grave de la calidad de vida. Otra causa de la estenosis esofágica durante la niñez es el desarrollo luego de una anastomosis término-terminal en los niños sometidos a cirugía por atresia esofágica. En general, este tipo de estenosis se produce en la porción proximal y media del esófago.

El protocolo de tratamiento para las quemaduras del esófago por cáusticos en nuestra institución incluye la evaluación inicial con esofagoscopía de control seguida de tratamiento con antibióticos, antihistamínicos $\mathrm{H}_{2}$ y corticoesteroides y conservación de la alimentación con sonda nasogástrica. Luego, se seleccionaron programas de dilatación con globo según la clasificación de la estenosis y la condición clínica del paciente.

El intervalo entre las dilataciones se establece según los resultados de las dilataciones previas y los síntomas clínicos. La dilatación con globo por radioscopía se describió por primera vez en Londres en 1981 y se aceptó como modalidad adecuada de tratamiento para la estenosis producida por quemaduras del esófago por cáusticos. ${ }^{7}$ Se ha informado que el éxito del tratamiento de la estenosis esofágica por cáusticos depende del manejo temprano y la selección correcta de los programas de dilatación. ${ }^{8}$

Por este motivo, muchas veces los pacientes reciben anestesia. El programa de dilatación se considera satisfactorio cuando se mantiene la disfagia en grado 0 o 1 durante 12 meses después del último procedimiento de dilatación. ${ }^{9}$

Serhal y col., informaron una tasa del $37 \%$ de desarrollo de estenosis en 62 pacientes tratados y dicho desarrollo fue de acuerdo con la tensión de la anastomosis. ${ }^{10}$

Se observó un aumento de los niveles de PIM en nuestros pacientes de ambos grupos debido a que se requiere insuflación durante los procedimientos endoscópicos en el esófago. En el Grupo ED, la PIM aumentó significativamente durante la dilatación como resultado de la tensión sobre la tráquea causada por la presión del globo. El aumento de la PIM fue mayor en los pacientes con estenosis proximal que en aquellos con estenosis media distal. Dado que el procedimiento de dilatación afecta los parámetros respiratorios y el volumen corriente disminuye

Tabla 5. Parámetros hemodinámicos

\begin{tabular}{|c|c|c|c|c|}
\hline Grupo E & $\begin{array}{c}\mathbf{A E} \\
\text { media } \pm \mathrm{DE}\end{array}$ & & $\begin{array}{c}\mathrm{DE} \\
\text { media } \pm \mathrm{DE}\end{array}$ & Valor $p$ \\
\hline PAS & $106,4 \pm 18,0$ & & $104,6 \pm 14,55$ & 0,06 \\
\hline PAD & $58,8 \pm 10,8$ & & $56,0 \pm 8,3$ & 0,270 \\
\hline FC & $114,4 \pm 15,7$ & & $112,5 \pm 15,1$ & 0,054 \\
\hline Grupo ED & $\begin{array}{c}\mathrm{AE} \\
\text { media } \pm \mathrm{DE}\end{array}$ & D & $\begin{array}{c}\mathrm{DE} \\
\text { media } \pm \mathrm{DE}\end{array}$ & Valor $p$ \\
\hline$\overline{\text { PAS }}$ & $97,1 \pm 16,0$ & $93,7 \pm 17,9$ & $94,5 \pm 12,7$ & 0,089 \\
\hline PAD & $55,2 \pm 10,1$ & $54,1 \pm 12,1$ & $55,6 \pm 8,8$ & 0,608 \\
\hline FC & $122,2 \pm 11,8$ & $132,0 \pm 13,1$ & $117,1 \pm 10,7$ & $<0,001$ \\
\hline
\end{tabular}

Los datos se expresan como media $\pm \mathrm{DE}$.

D: dilatación. AE: antes de la endoscopía. DE: después de la endoscopía. PAS: presión arterial sistólica.

PAD: presión arterial diastólica. FC: frecuencia cardíaca. 
como resultado de la tensión, se eleva la Pinsp para mantener el volumen corriente adecuado. En cinco pacientes menores de 2 años, la presión de las vías respiratorias aumentó drásticamente, fue imposible continuar con la ventilación y se inició la desaturación.

El aumento de la PIM como resultado de la presión del globo durante la dilatación fue mayor en los niños pequeños según el nivel de madurez de la tráquea conforme a la edad. Disminuir la presión del globo permitió mantener la ventilación en estos pacientes.

La dilatación gástrica como resultado de la insuflación de aire durante la endoscopía disminuye la distensibilidad pulmonar, en particular en los niños más pequeños. Esta condición aumenta significativamente la PIM en los niños menores de 2 años con respecto al valor inicial en ambos grupos.

Con frecuencia se observan tos persistente, ronquera, hiperreactividad de las vías respiratorias, neumonitis recurrente como resultado de la inflamación crónica de las vías respiratorias y los pulmones debido a la dificultad para tragar la saliva en los niños con estenosis grave. La tos persistente, la agitación, las náuseas, los vómitos y las alteraciones de la voz fueron complicaciones frecuentes en el grupo con dilatación y estenosis en nuestro estudio. La continuidad de los problemas crónicos de las vías respiratorias puede provocar complicaciones respiratorias obstructivas y restrictivas en estos niños.

No se observaron cambios en la PAS, la PAD y la FC de los pacientes del Grupo E durante la endoscopía. La PAS y la PAD se mantuvieron estables durante la dilatación en los pacientes del Grupo ED. La FC aumentó durante el procedimiento en comparación con el estado inicial y disminuyó después de la dilatación. Las complicaciones observadas en el Grupo ED fueron disminución repentina de la FC en cuatro pacientes y bradicardia que requirió intervención en uno. Las fibras sensoriales principales del esófago llegan a los nervios vago y raquídeos. Los cambios hemodinámicos resultantes de la tensión mecánica sobre la pared del esófago podrían relacionarse con la teoría de que las fibras aferentes del corazón y el esófago se encuentran en las mismas neuronas de la vía espinotalámica. ${ }^{4}$

La dilatación del esófago con globo podría provocar complicaciones respiratorias y cardiovasculares debido a la tensión mecánica del globo sobre la superficie y las vías respiratorias y los mecanismos reflejos. Se ha demostrado que la distensión esofágica modifica los patrones ventilatorios y la distribución de la actividad motora en los músculos respiratorios a modo de reflejo. ${ }^{4}$ Por lo tanto, dicho reflejo puede ser un problema en los procedimientos de dilatación con sedación. Jacobsen y col., observaron las siguientes complicaciones durante la insuflación del globo: cuatro de cada diez pacientes necesitaron intervención médica para lograr una función cardiovascular adecuada, cuatro pacientes desarrollaron taquicardia y tres tuvieron desviaciones del segmento ST. ${ }^{11}$

Por el contrario, en la serie de Gerçek y col., la presión arterial aumentó en todos los pacientes excepto uno, la FC se incrementó significativamente y se revirtió cuando se desinfló el globo, como en nuestros pacientes. Los autores informaron que no fue posible mantener la ventilación en cuatro pacientes debido a una hemorragia. También observaron obstrucción distal del tubo endotraqueal en dos pacientes, extubación durante el procedimiento en un paciente y broncoespasmo tras la extubación en dos pacientes.

La dilatación del esófago con globo es una intervención con alto riesgo de complicaciones según lo informado en la bibliografía y los hallazgos de nuestra serie. Es necesario seguir atentamente la presión del globo y de las vías respiratorias y las alteraciones en el volumen corriente. Se debe tener en cuenta que la extubación puede producirse durante la dilatación y todo el procedimiento debido a los efectos mecánicos. En uno de nuestros pacientes también se produjo la extubación durante el procedimiento de dilatación.

La dilatación esofágica puede causar complicaciones graves, como perforación, hemorragia y aspiración. La perforación se caracteriza por dolor agudo, disnea, taquicardia y fiebre. En un estudio previo en nuestra institución se observaron lesiones esofágicas en cuatro pacientes; sin embargo, ninguno presentó lesiones de rotura durante el período del estudio. ${ }^{12}$

Son fundamentales el uso de anestesia general y la intubación para las intervenciones riesgosas que podrían causar tensión mecánica. Además, la anestesia general permite lograr un entorno de trabajo seguro y cómodo para el equipo quirúrgico a cargo de la dilatación. Es posible que las complicaciones estén asociadas con la intensidad de la presión del globo y el grado y la extensión de la estenosis esofágica; sin embargo, 
para indicar dicha asociación, es necesario realizar una evaluación prospectiva de una gran cantidad de pacientes con una clara definición de la ubicación y la extensión de la estenosis.

Asimismo, la desnutrición causada por una alimentación inadecuada, la inflamación de las vías respiratorias y los pulmones como resultado de la dificultad para tragar saliva y la aspiración frecuente complican el uso de anestesia en estos pacientes.

\section{CONCLUSIÓN}

La presión en las vías respiratorias aumentó en los pacientes de ambos grupos durante la endoscopía, en especial entre los de menor edad y de manera más pronunciada durante la dilatación con globo y en los pacientes con estenosis esofágica proximal. En el grupo con dilatación, se detectó un aumento estadísticamente significativo de la FR y se observó la aparición repentina de bradicardia en un paciente durante el procedimiento de dilatación. Las complicaciones posoperatorias como la tos, la agitación, la taquicardia, las náuseas y los vómitos y la alteración de la voz fueron frecuentes en el grupo con dilatación con globo.

Es necesario observar atentamente y tratar cuidadosamente las complicaciones respiratorias y cardiovasculares graves que pueden ocurrir durante la dilatación con globo y con anestesia general.

\section{REFERENCIAS}

1. Huang YC, Ni YH, Lai HS, et al. Corrosive esophagitis in children. Pediatr Surg Int 2004;20(3):207-10.

2. Faccin G,MerloF, Moretti T, etal. Anesthesiologic problems in transluminal balloon dilatation of esophageal stenosis in children. Minerva Anesthesiol 1990;56(3):77-80.

3. Zubair NA, Patil DR, Alghamdi AH. A rare and fatal complication of oesophagoscopy. Anaestheticimplications. Anaesthesia 1994;49(10):873-5.

4. Gerçek A, Ay B, Doğan V, et al. Esophageal balloon dilation in children: prospective analysis of hemodynamic changes and complications during general anesthesia. J Clin Anesth 2007;19(4):286-9.

5. RiffatF, Cheng A. Pediatric causticingestion: 50 consecutive cases and a review of the literature. Dis Esophagus 2009;22(1):89-94.

6. Doğan Y, Erkan T, Çokuğraş FC, et al. Caustic gastroesophageal lesions in childhood: An analysis of 473 cases. Clin Pediatr (Phila) 2006;45(5):435-8.

7. London RL, Trotman BW, DiMarino AJ Jr, et al. Dilatation of severe esophageal strictures by an inflatable balloon catheter. Gastroenterology 1981;80(1):173-5.

8. Uygun I, Arslan MS, Aydoğdu B, etal. Fluoroscopic balloon dilatation for caustic esophageal strictur in children: an 8 year experience. J Pediatr Surg 2013;48(11):2230-4.

9. Cabrera Chamorro C, Méndez MancholaC, Molina Ramirez I, et al. Endoscopic balloon dilatation of esophageal strictures in children. Cir Pediatr 2013;26(3):106-11.

10. Serhal L, Gottrand F, Sfeir F, et al. Anastomotic stricture after surgical repair of esophageal atresia: frequency, risk factors, and efficacy of esophageal bougie dilatations. J Pediatr Surg 2010;45(7):1459-62.

11. Jakobsen JH, Rasmussen V, Rosenberg J. Cardiovascular effects of oesephageal dilation under general anaesthesia. Dan Med Bull 1999;46(3):252-4.

12. Erdoğan E, Eroğlu E, Tekant G, et al. Management of esophagogastric corrosive injuries in children. Eur J pediatr Surg 2003;13(5):289-93.

\section{Corrección}

Los autores del artículo original "Evaluación del manejo del dolor de la patología facial y oral en un Servicio de Urgencias Pediátrico y tras el alta domiciliaria" publicado en Arch Argent Pediatr 2018;116(1):28-34 informan que han cometido errores en los apellidos de dos de los autores.

En la versión impresa, donde dice Gloria Guerrero López debe decir Gloria Guerrero Márquez y donde dice Paula López Vázquez debe decir Paula Vázquez López. 